\title{
Alimentation complémentaire du réservoir de Naussac dans le bassin du Haut-Allier
}

\author{
Jean Clérin, Directeur, Département Hydraulique SOMIVAL \\ Roland Duranton, Conseiller Technique, Département Hydraulique SOMIVAL \\ Régis Thépot, Adjoint Directeur de l'E.P.A.L.A.
}

\section{Généralités}

\subsection{Présentation}

Le bassin ligérien s'étend sur $117000 \mathrm{~km}^{2}$ et occupe ainsi $21 \%$ du territoire national. La Loire y prend sa source en Ardèche dans les monts du Vivarais à plus de $1500 \mathrm{~m}$ d'altitude. Elle rejoint l'océan à Saint-Nazaire après un cours total estimé à $1070 \mathrm{~km}$. L'Allier prend sa source à $1473 \mathrm{~m}$, au Moure de la Gardille en Lozère. Elle se jette dans la Loire au Bec d'Allier après $410 \mathrm{~km}$ en cours (BV $14300 \mathrm{~km}^{2}$ ).

Le bassin de la Loire se distingue des grandes unités hydrographiques françaises par des particularités marquées quant à la nature géologique des terrains et à son régime hydrologique.

Partagé entre les zones hercyniennes, où granits et gneiss donnent par érosion des terrains acides et imperméables, et les régions volcaniques rencontrées en Haute-Loire, dans le Puy-de-Dôme et le Cantal, le Massif Central reçoit les plus fortes précipitations du bassin ligérien. Le régime de la Loire elle-même, et de son principal affluent de l'Allier, se trouve ainsi très intimement lié au climat de cette région qui l'influence à l'aval jusque vers les confluents du Cher et de la Vienne.

Ainsi, ces rivières, à la sortie du Massif Central, connaissent une très grande irrégularité dans leurs apports. L'examen des séries de débit permet de juger, après plus d'un demi-siècle d'observations, de la violence de certaines crues mais aussi de la faiblesse fréquente des étiages. A titre d'exemple, le débit naturel annuel de l'Allier à VieilleBrioude (BV $2270 \mathrm{~km}^{2}$ ), en fréquence décennale, est égal à la moitié de sa valeur moyenne $\left(30 \mathrm{~m}^{3} / \mathrm{s}\right)$ avec des étiages mensuels de moins de $2 \mathrm{~m}^{3} / \mathrm{s}$, alors que les crues peuvent y être de l'ordre de $2000 \mathrm{~m}^{3} / \mathrm{s}$.

En fait dans leurs bassins supérieurs Loire et Allier subissent l'influence cévenole voisine qui contribue à cette irrégularité très marquée du régime.

Or, l'Allier traverse des zones agricoles très riches notamment les bassins d'effondrement comblés d'alluvions qui constituent les Limagnes. La grande incertitude pesant sur la ressource en eau dans cette zone de l'Auvergne a limité son essor économique. L'irrigation, permettant l'amélioration, la diversification et la régularité des productions agricoles, ainsi qu'en conséquence l'augmentation du potentiel industriel régional, n'a pu connaître un développement normal qu'après la création de la retenue de Naussac sur le haut bassin destinée à y stocker l'eau en période d'abondance pour la restituer suivant les besoins en étiage.

Parmi les sites favorables à la construction de barrages sur le Haut-Allier et ses affluents, Naussac était le plus intéressant. Grâce à une digue sur le Donozau en Lozère, dont la hauteur au-dessus du terrain naturel est de $50 \mathrm{~m}$ et la longueur en crête de $240 \mathrm{~m}$, il a été possible d'obtenir une retenue de $190 \mathrm{Mm}^{3}$ pour un plan d'eau de 1050 ha à 945 NGF. Un barrage moins haut, $40 \mathrm{~m}$ par exemple, aurait eu une capacité de $100 \mathrm{Mm}^{3}$ pour une superficie de 750 ha à $940 \mathrm{NGF}$, ce qui est apparu moins intéressant économiquement et pour les besoins prévisibles.

\subsection{Historique}

Les qualités remarquables du site de Naussac ont été d'abord prises en considération par les hydroélectriciens qui avaient vu l'intérêt de ce réservoir comme pièce maîtresse du projet d'aménagement complémentaire dit de " Montpezat $\mathrm{C}$ » dont la demande de concession fut déposée par EDF en 1952, avec une cote de retenue normale du barrage de Naussac à 942 NGF.

Dans ce projet, la retenue de Naussac concentrait les apports du Haut-Allier par deux adductions, l'une en provenance de la branche Chapeauroux comprenant Ance du Sud, Grandrieu et Chapeauroux, l'autre de la branche Allier comprenant Espézonnette, Langouyrou et Allier. Ces eaux devaient être turbinées dans une usine dite de Naussac. Puis, à partir du barrage de Saint-Etienne-duVigan sur l'Allier, elles étaient envoyées par galerie dans le réservoir d'Arlempdes sur la Loire d'où une galerie parallèle à celle de Montpezat préexistante alimentait directement l'usine de Montpezat C sur la Fontaulière, affluent de l'Ardèche. 


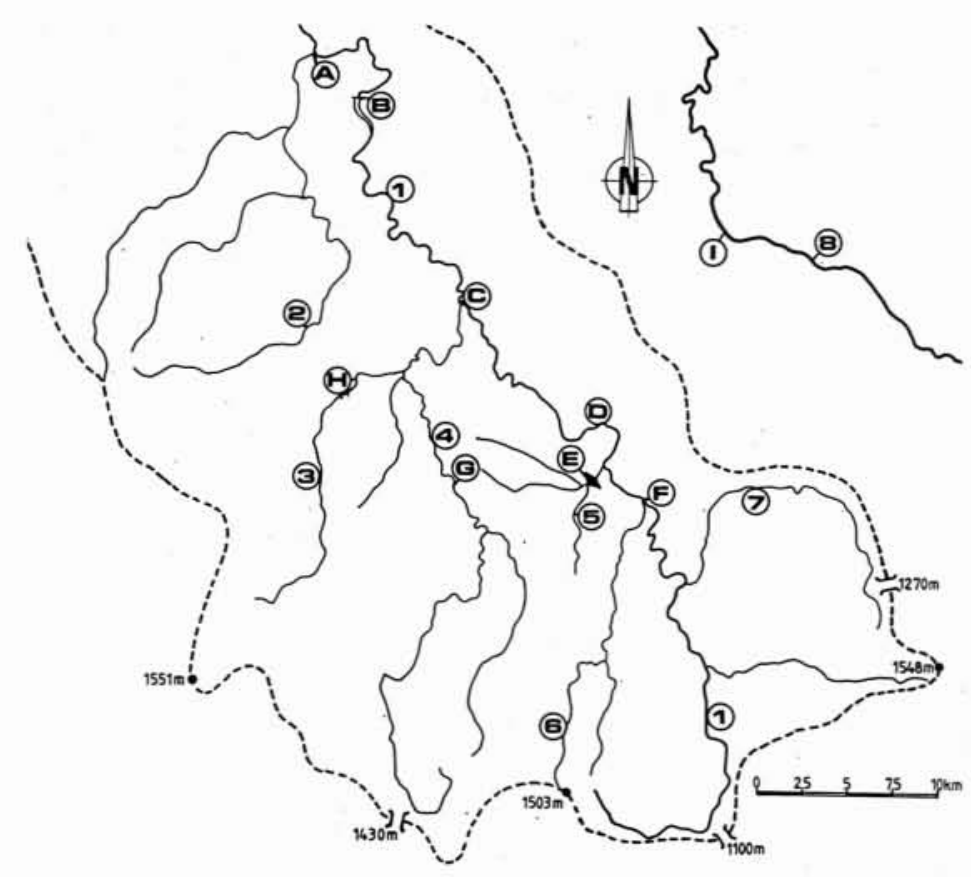

Rivières (rivers)

1 - Allier

2 - Ance du Sud

3- Grandrieu

4- Chapeauroux

5 - Donozau

6 - Langouyrou

7 - Espézonnette

8 - Loire

A - MONISTROL

B - POUTES (barrage)

C - CHAPEAUROUX

D - ST-ÉTIENNE DU VIGAN (barrage)

E - NAUSSAC (barrage)

F - LANGOGNE

G - AUROUX

H - GRANDRIEU

I - ARLEMPDES

1. Haut-Allier - Réseau hydrographique.

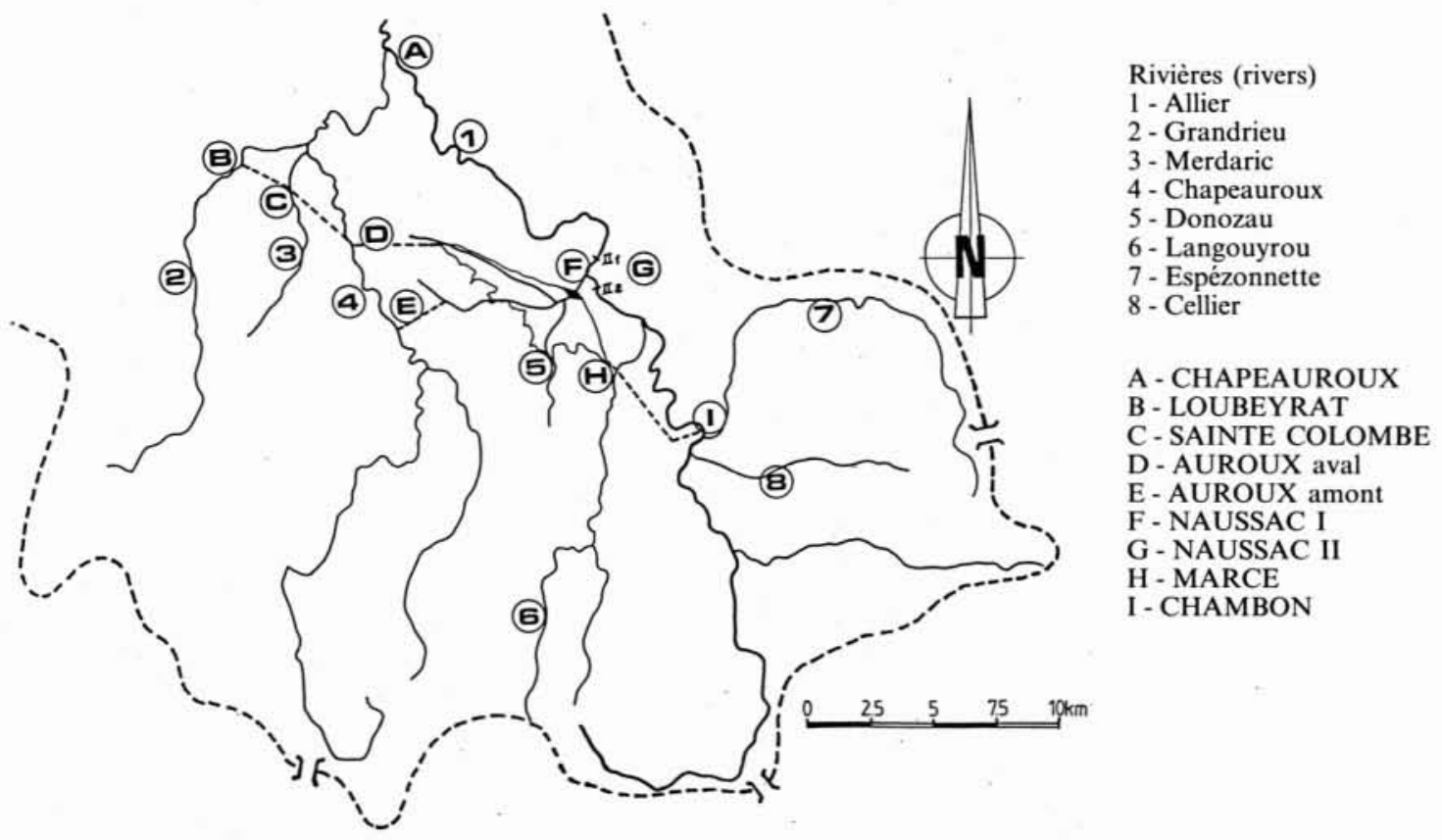

2. Dérivations gravitaires. 
Ce projet dut finalement être abandonné en raison des réticences suscitées par le changement de bassin versant Loire-Rhône des apports de la Loire et de l'Allier supérieurs, et la réduction correspondante des débits disponibles dans ces rivières à l'aval.

Par la suite, vers 1970, l'Etat a repris cette affaire et chargé SOMIVAL par l'intermédiaire du Ministère de l'Agriculture, d'étudier dans quelle mesure le site de Naussac pourrait être utilisé pour créer un réservoir destiné à alimenter en eau lors des périodes d'étiages le Val d'Allier ainsi que le Val-de-Loire à l'aval du Bec d'Allier.

\section{Inventaire des ressources}

\subsection{Le Donozau}

Si le site de Naussac permettait de réaliser un réservoir de grande capacité dans des conditions optimales, l'alimentation de la retenue ne pouvait être assurée par le ruisseau du Donozau dont l'apport moyen annuel est de $20 \mathrm{Mm}^{3}$ environ pour un bassin versant de $55 \mathrm{~km}^{2}$.

\subsection{Les affluents de l'Allier}

Il fallait donc prévoir une alimentation complémentaire à partir de rivières voisines. Diverses adductions gravitaires ont été envisagées sur les rivières Chapeauroux, Grandrieu, Allier, Espezonnette et Langouyrou contrôlant globalement, en amont des points de prises envisagés, un bassin versant de $700 \mathrm{~km}^{2}$ (fig. 1).

Ce bassin versant est délimité à l'ouest par les crêtes de la Margeride, au sud par les Cévennes et à l'est par les collines entre Loire et Allier qui s'appuient sur les monts du Velay. La ligne de crête qui entoure vers $1500 \mathrm{~m}$ cet amphithéâtre s'abaisse le long de trois seuils:

- seuil de Montbel (1 $230 \mathrm{~m})$, ouvert aux influences atlantiques,
- seuil de La Bastide (1 $100 \mathrm{~m})$, ouvert aux influences méditerranéennes,

- seuil entre Ardèche et Allier (1 $270 \mathrm{~m}$ ), ouvert aux influences rhodaniennes.

Les apports possibles au barrage de Naussac (période 1946 à 1967) ont été établis avec des débits journaliers aux prises, reconstitués par corrélation à partir des données fournies par les stations hydrométriques les plus proches (7 sur le bassin étudié, 3 à l'extérieur).

Les apports moyens annuels disponibles sont de l'ordre de $350 \mathrm{Mm}^{3}$, déduction faite des débits de franchise, c'està-dire satisfaisants pour l'alimentation du réservoir.

\subsection{Alimentations gravitaires de la retenue}

Le prédimensionnement des dérivations gravitaires visait à prélever au minimum $80 \%$ de l'Allier et plus de $90 \%$ du Chapeauroux (fig. 2).

Les faibles marges d'altitude entre points de prise et retenue de Naussac n'autorisaient pas de dérivation gravitaire quelle que soit la cote du plan d'eau : cet inconvénient était dû à la cote de retenue normale du réservoir, surélevée de $3 \mathrm{~m}$ par rapport au projet EDF.

En particulier sur l'Allier, pour ne pas perdre les apports du Cellier et de l'Espezonnette, il aurait fallu dériver leurs débits par une conduite complémentaire ou alors refouler l'eau à partir d'une certaine cote de retenue. En tout cas cette solution se révélait coûteuse et aléatoire.

Par ailleurs les prises d'eau multiples nécessaires réduisent les apports utiles pour le réservoir par suite des débits réservés et dérivés. L'exploitation est plus lourde et complexe. La protection de l'environnement local est maltraitée. Quant à l'aspect apports gravitaires - énergie hydroélectrique, il reste relativement marginal: l'intérêt économique d'une centrale à construire spécialement n'est pas évident avec la part d'énergie d'été produite et les contraintes imposées à l'exploitant pour limiter les nuisances.

\begin{tabular}{|c|c|c|c|c|}
\hline Rivière & Point de prise & $\begin{array}{c}\text { Galerie } \\
\varnothing(\mathrm{m}), \mathrm{L}(\mathrm{km})\end{array}$ & $\begin{array}{l}\text { Débit maximum } \\
\mathrm{m}^{3} / \mathrm{s}\end{array}$ & Remarques \\
\hline Donozau & Naussac & - & - & \\
\hline Allier & $\begin{array}{l}\text { confluent } \\
\text { Espézonnette }\end{array}$ & $4,5-5$ & 20 & \\
\hline Langouyrou & Marcé & $4,5-1$ & 5 & + reprise de I'Allier \\
\hline Chapeauroux & amont Auroux & $2,5-3$ & 1,5 & \\
\hline Chapeauroux & aval Auroux & $3 / 4-4$ & $8 / 15$ & $\begin{array}{c}+ \text { reprise du } \\
\text { Grandrieu }\end{array}$ \\
\hline Gandrieu & Grandrieu & $2-9$ & 6 & \\
\hline
\end{tabular}


Finalement seule l'adduction du Chapeauroux avec prise à l'amont d'Auroux a été adoptée comme la plus directe et la moins onéreuse : si elle ne permet pas d'adjoindre par raccordement la dérivation du Grandrieu et du Merdaric, il n'y a pas d'inconvénient puisque l'opposition locale à une telle dérivation a été dès cette époque permanente et absolue pour exiger des responsables de l'opération l'engagement irrévocable de l'exclure de l'aménagement.

Les apports naturels correspondants du Chapeauroux représentent environ $80 \mathrm{Mm}^{3}$ en moyenne annuelle.

\subsection{Pompage dans I'Allier}

Compte tenu des inconvénients majeurs présentés par une solution gravitaire il est apparu préférable de concevoir une alimentation plus simple du réservoir.

Les déficits de remplissage constatés sur la série d'années sèches 1946/1953 — il en serait de même sur 1989-1991 — ne pouvaient être comblés que par un pompage complémentaire dans l'Allier au niveau du hameau de La Valette près du confluent Donozau-Allier. Bien qu'à priori quelques doutes pourraient être émis sur la rentabilité de l'installation de pompage destinée à ne fonctionner qu'épisodiquement, celle-ci assure en réalité la garantie d'alimentation du réservoir puisque ce sont justement pendant les années sèches que la valeur d'usage de l'eau est maximale.

Il faut préciser pour la branche principale Allier que le bassin versant capté par pompage est supérieur de $140 \mathrm{~km}^{2}$ à celui disponible dans la solution gravitaire globale, où en contrepartie la branche Chapeauroux avec prise à l'aval d'Auroux dans cette solution majore le bassin versant dérivé de $15 \mathrm{~km}^{2}$ environ.

\section{Aménagement de Naussac}

L'analyse des diverses possibilités d'alimentation de la retenue et du fonctionnement du barrage a finalement conduit à retenir la réalisation de l'alimentation en deux phases (CIANE du 26 juillet 1973), la première incluant le Donozau et la dérivation du Chapeauroux, la deuxième prévoyant l'alimentation complémentaire ultérieure du réservoir par pompage dans l'Allier. Néanmoins, il fut décidé de construire le barrage lui-même directement à sa capacité définitive dès la première phase.

Ainsi fut établi dès l'origine le programme complet de la réalisation de l'aménagement de Naussac en deux étapes.

\subsection{Naussac I}

Après enquêtes, la première phase d'aménagement du réservoir de Naussac d'une capacité de $190 \mathrm{Mm}^{3}$ destiné à régulariser les débits de l'Allier et de la Loire a été déclarée d'utilité publique par décret interministériel du 6 février 1976.
Les opérations d'aménagement hydrauliques comprenaient :

- la construction d'une digue à la cote 947 NGF sur le Donozau ;

- la construction des digues complémentaires du Cheylaret (digue de col) et du Mas d'Armand (plan d'eau à niveau constant) ;

- l'établissement des ouvrages de dérivation des eaux du Chapeauroux.

Par décret du 11 juin 1976, l'aménagement et l'exploitation du réservoir de Naussac étaient concédés à SOMIVAL.

Les travaux des ouvrages de première phase ont été entrepris en avril 1977 et se sont terminés en avril 1981. Le premier remplissage du réservoir commencé fin octobre 1980 s'est achevé en novembre 1982.

Depuis 1983, l'ouvrage permet de satisfaire l'objectif réglementaire de $6 \mathrm{~m}^{3} / \mathrm{s}$ à Vieille-Brioude et de participer avec le réservoir de Villerest à l'objectif de gestion situé à l'entrée de la Loire moyenne à Gien. C'est en moyenne $70 \mathrm{Mm}^{3}$ (variable entre 28 et $125 \mathrm{Mm}^{3}$ ) qui ont été déstockés au cours des neuf années d'exploitation, mais en freinant les déstockages lors des années très sèches afin de limiter autant que possible le déficit au remplissage l'année suivante (cf. 1989-90-91) faute d'être assuré d'apports suffisants en l'absence des installations de pompage.

\subsection{Naussac II}

Les travaux d'aménagement de deuxième phase devaient s'enchaîner avec ceux de la première phase. Ils comprenaient :

- un ouvrage de prise sur l'Allier à La Valette,

- une station de pompage-turbinage en pied de barrage pour refoulement dans le réservoir et production annexe d'énergie au moment des lâchures.

L'ouvrage de prise sur l'Allier consistait en un barrage mobile d'une hauteur de $19 \mathrm{~m}$ sur fondation, équipé de 2 vannes segments capables d'évacuer la crue millenale de $1700 \mathrm{~m}^{3} / \mathrm{s}$. Une retenue d'une capacité utile de $1,2 \mathrm{Mm}^{3}$ était ainsi constituée au pied du barrage de Naussac. Une petite centrale hydroélectrique annexe était incorporée en rive gauche du barrage sur l'Allier avec un débit équipé de $28 \mathrm{~m}^{3} / \mathrm{s}$ et une puissance installée de $3 \mathrm{MW}$.

L'usine de pompage turbinage en puits, située au pied aval du barrage de Naussac, était équipée de 2 groupes réversibles Francis d'un débit maximal de $2 \times 24 \mathrm{M}^{3} / \mathrm{s}$ en pompe et $2 \times 20 \mathrm{~m}^{3} / \mathrm{s}$ en turbine correspondant à une puissance installée respectivement de $26 \mathrm{MW}$ et $20 \mathrm{MW}$.

Le refoulement des débits pompés est naturellement assuré par la conduite de restitution et de vidange existante, placée sous le barrage de Naussac en première phase.

Accessoirement il était prévu sur la dérivation du Chapeauroux l'équipement d'une autre petite centrale hydraulique d'un débit de $6 \mathrm{~m}^{3} / \mathrm{s}$ et d'une puissance installée de $2 \mathrm{MW}$ pour une chute brute maximale de $43 \mathrm{~m}$.

Le petit réservoir sur l'Allier jouait le rôle de bassin tampon, au pompage et au turbinage, car les groupes 
n'étaient pas réglables. Ce bassin permettait aussi le transfert d'énergie journalier heures pleines/heures creuses avec le réservoir principal amont, ce qui permettait une meilleure rentabilité de l'investissement par revalorisation de l'énergie.

La demande de concession de force hydraulique de la chute a été présentée par SOMIVAL fin 1982. Après instruction administrative et enquêtes publiques, l'Etat a décidé en 1985, avant transmission de la demande au Conseil d'Etat, de ne pas lui donner suite.

\section{Reprise de Naussac II}

L'Etablissement Public d'Aménagement de la Loire et de ses Affluents (E.P.A.L.A.) ayant notamment pour objet le soutien des étiages a repris à son compte le projet Naussac II repoussé. Par convention, il a donné mandat à SOMIVAL pour sa réalisation.

L'actualisation du projet a incité à examiner si compte tenu de son impact la production énergétique restait intéressante économiquement ainsi que le transfert d'énergie journalier.
La recherche d'impact moindre sur l'environnement a conduit à retenir des machines spéciales fonctionnant à débit et hauteur réglables afin de réduire le barrage de prise sur l'Allier qui malgré sa taille modeste présentait un obstacle à l'écoulement (fig. 3) avec certaines submersions.

\subsection{Le nouveau projet}

Les modifications suivantes ont ainsi été apportées :

- l'ouvrage sur l'Allier est situé plus en amont, avant le confluent avec le Donozau, et se réduit à un seuil de 2,3 m de hauteur. L'ouvrage de franchissement pour les poissons est simplifié. Il n'existe plus de chute équipable à $\mathrm{La}$ Valette par une petite centrale hydroélectrique comme c'était le cas sur le barrage de prise prévu antérieurement pour le premier projet de Naussac II ;

- un chenal d'amenée suivi d'une galerie de $340 \mathrm{~m}$ permettent le transfert des eaux de l'Allier dans le bassin de pompage créé sur le Donozau canalisé et fermé à son débouché aval par un vannage;

- les deux pompes turbines Francis sont remplacées par trois machines hydrauliques Deriaz réversibles de chacune

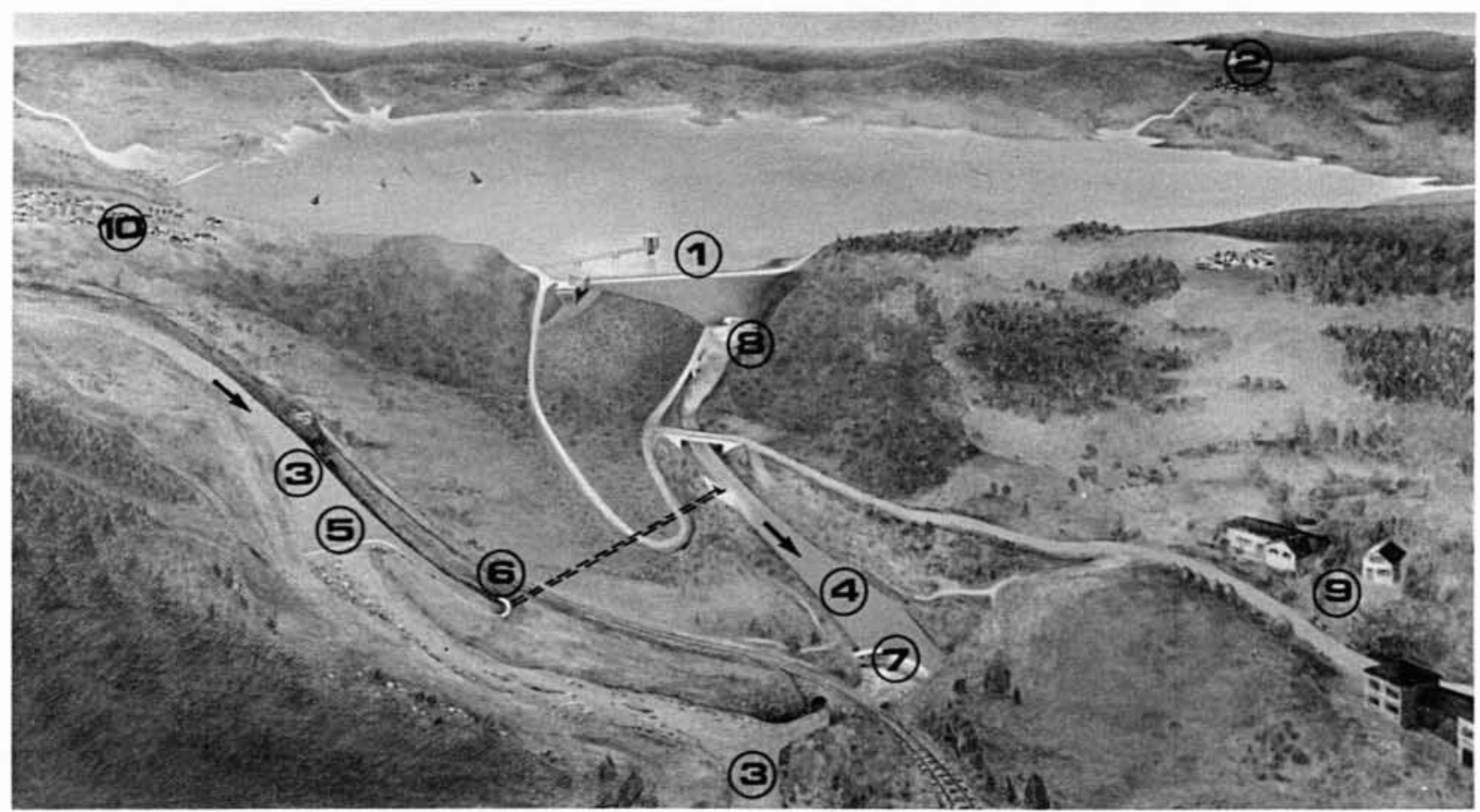

1- Barrage de NAUSSAC

2 - Dérivation du Chapeauroux

3 - Allier

4- Donozau

5 - Seuil sur l'Allier
6 - Chenal et galerie

7 - Seuil sur Donozau

8 - Usine de pompage turbinage

9 - LAVALETTE

10 - NAUSSAC

3. Aménagement de Naussac. 


\section{J. CLÉRIN et al.}

$3 \mathrm{MW}$ en pompe et 2,65 MW en turbine avec $56 \mathrm{~m}$ de chute brute maximale et $5 \mathrm{~m}^{3} / \mathrm{s}$ de débit maximal unitaire. Elles fonctionnent pratiquement au fil de l'eau. Les dimensions de la centrale sont réduites, la structure restant inchangée. La faculté de transfert d'énergie est supprimée, son intérêt étant très réduit. Les conditions de démarrage des groupes sont améliorées. L'utilisation du tarif EDF Effacement Jours de Pointe (E.J.P.) autorise quand c'est nécessaire un pompage quasi continu à moindre coût, sauf insuffisance des apports disponibles dans l'Allier.

\subsection{Simulations de remplissage}

Les simulations de remplissage ont été effectuées sur les 44 dernières années (1946-1989). Une courbe d'alerte correspondant à un remplissage à $90 \%$ au $1^{\text {er }}$ juin a été établie. C'est à partir de cette courbe que le pompage est mis en marche dans les simulations.

Sur les 44 années, on ne pompe en fait que 19 années. Lors de ces années, le pompage a lieu surtout en mars et avril ( $60 \%$ du volume moyen annuel pompé). En année décennale sèche, le volume annuel pompé dépasse $57 \mathrm{Mm}^{3}$.
Il s'agit des années où Naussac II est absolument indispensable pour remplir le réservoir et pour lesquelles le soutien d'étiage est particulièrement crucial. Dans ce cas, on pourra disposer de $170 \mathrm{Mm}^{3}$ début juillet contre $111 \mathrm{Mm}^{3}$ actuellement.

La répercussion sur les lâchures, surtout en fréquences quinquennale et décennale, est importante, de 15 à $22 \%$ de plus en volume, permettant de mieux garantir les objectifs de débit sur l'Allier tout en améliorant les conditions hydrauliques du Chapeauroux qui verrait son débit réservé, fixé par décret lors de la D.U.P., passer de 0,3 à $0,6 \mathrm{~m}^{3} / \mathrm{s}$ (plus de $20 \%$ du module).

De même sur l'Allier, lors du pompage, le débit réservé retenu dans les simulations est de $2 \mathrm{~m}^{3} / \mathrm{s}$ soit plus de $20 \%$ du module.

\subsection{Réalisation de Naussac II}

La demande de concession de force hydraulique est en cours d'établissement. A l'issue des procédures administratives, la réalisation des ouvrages et la mise en service nécessiteront deux à trois années. 